\title{
DIAGNOSTIC UTILITY OF AMACR IN PROSTATIC CARCINOMA
}

\author{
${ }^{1}$ Senior Resident, Department of Pathology, Government Medical College, Kottayam. \\ 2Professor, Department of Pathology, Government Medical College, Kottayam. \\ 3 Professor, Department of Pathology, Government Medical College, Kottayam. \\ ${ }^{4}$ Professor, Department of Pathology, Government Medical College, Kottayam. \\ ${ }^{5}$ Associate Professor, Department of Pathology, Government Medical College, Kottayam. \\ ${ }^{6}$ Assistant Professor, Department of Pathology, Government Medical College, Kottayam. \\ ${ }^{7}$ Assistant Professor, Department of Pathology, Government Medical College, Kottayam.
}

Sangeetha Kandasamy1, Lillykutty Pothen ${ }^{2}$, Letha V3, Sankar S4, Priya P. V5, Sheela Varghese ${ }^{6}$, Jayalakshmy P. $L^{7}$

ABSTRACT

\section{BACKGROUND}

The aim of the study is to assess the sensitivity and specificity of AMACR (P504S) in the diagnosis of prostatic carcinoma and to correlate serum PSA value in Carcinoma Prostate, atypia, PIN, prostatitis and nodular hyperplasia. This was an observational descriptive study carried out in Department of Pathology, Government Medical College, Kottayam.

\section{MATERIALS AND METHODS}

Total of 120 cases of prostatic needle biopsies (processed routinely for $\mathrm{H}$ and $\mathrm{E}$ and IHC) during the period of 18 months from August 2014 to January 2016 were studied. Statistical analysis was done using SPSS version 16. Chi- square test was used to find if there was any statistically significant association between the levels of serum PSA and histopathological findings studied.

\section{RESULTS}

Among 93/120 cases confirmed as carcinoma by H and E, 85 cases (91.3\%) showed AMACR positivity. Among 5/120 cases confirmed as atypical glands suspicious for malignancy by H and E, 4 cases (80\%) showed AMACR positivity. Out of 3/120 cases confirmed as PIN by H and E, 2 cases (66\%) showed AMACR positivity. Out of 19/120 cases confirmed as benign prostatic hyperplasia by $\mathrm{H}$ and $\mathrm{E}$, all the 19 cases (100\%) showed AMACR negativity. High grade carcinoma (Gleason pattern 5) showed AMACR negativity. Sensitivity and specificity of AMACR in prostatic carcinoma were $90 \%$ and $100 \%$ respectively. Positive predictive value of AMACR in prostatic carcinoma was 100\% and negative predictive value of AMACR in prostatic carcinoma was $65 \%$; $99 \%$ of prostatic carcinoma showed high serum PSA value.

\section{CONCLUSION}

$\mathrm{H}$ and $\mathrm{E}$ is the gold standard for diagnosing prostatic neoplasia. AMACR helps in confirming the diagnosis of carcinoma in the cases of atypical glands/suspicious of malignancy. But AMACR negativity does not exclude the possibility of carcinoma. So along with positive marker (AMACR), basal cell marker (negative marker, p63/HMWCK) is useful in diagnosing the morphologically suspicious cases.

\section{KEYWORDS}

Prostatic Carcinoma, Gleason Pattern, AMACR

HOW TO CITE THIS ARTICLE: Kandasamy S, Pothen L, Letha V, et al. Diagnostic utility of AMACR in prostatic carcinoma. J. Evolution Med. Dent. Sci. 2017;6(35):2869-2873, DOI: 10.14260/Jemds/2017/619

\section{BACKGROUND}

The most common malignancy of prostate is adenocarcinoma, which accounts for more than $25 \%$ of all malignancies in men. ${ }^{1}$ In developing countries even though prostatic carcinoma is less common, its incidence and mortality is on the rise. ${ }^{2}$ It is a disease gaining importance worldwide. In Indian population, the incidence ranges from 5.39 to $6.58 / 1$ lakh population. ${ }^{3}$ Currently, the mainstay of detection of early prostatic carcinoma is by the triad of level of total serum PSA, digital rectal examination and TRUS-guided core needle

Financial or Other, Competing Interest: None.

Submission 23-03-2017, Peer Review 19-04-2017,

Acceptance 25-04-2017, Published 01-05-2017.

Corresponding Author:

Sangeetha Kandasamy,

Senior Resident,

C1 Block, Department of Pathology,

Government Medical College,

Gandhi Nagar, Kottayam-686008.

E-mail: sangeetharangs84@gmail.com

DOI: $10.14260 /$ jemds $/ 2017 / 619$

biopsy. ${ }^{4}$ The most commonly used cut-off value for serum PSA is $4 \mathrm{ng} / \mathrm{mL}$. In some cases of mimickers of carcinoma, a diagnosis of suspicious of atypical small acinar proliferation, but not diagnostic of malignancy has to be given. ${ }^{5}$ Due to the availability of limited tissue and presence of many benign mimickers of malignancy, diagnosis of prostatic carcinoma by $\mathrm{H}$ and $\mathrm{E}$ alone becomes difficult. Alpha-methylacyl-CoA racemase (AMACR), a recently discovered positive IHC marker for prostatic carcinoma is being increasingly used in combination with routine $\mathrm{H}$ and $\mathrm{E}$ staining and negative basal cell marker p63, especially in morphologically difficult needle biopsies of prostate. ${ }^{6}$ Therefore, this area offered an ideal research field. The present study is to find out diagnostic utility of AMACR in prostatic carcinoma, sensitivity and specificity of AMACR for detecting both benign and malignant prostatic lesions. In addition, serum PSA value is correlated with various prostatic lesions.

\section{MATERIALS AND METHODS}

After obtaining Ethical Committee approval, 120 cases of prostatic needle biopsies were analysed during the period of 
18 months from August 2014 to January 2016 in the Department of Pathology, Government Medical College, Kottayam, Kerala, India. The cases studied were benign prostatic tissue, prostatitis, atypical glands/suspicious for malignancy, PIN and carcinoma. In this study, transurethral resection of prostate and radical prostatectomy specimens were excluded. The prostatic biopsies were done in the Department of Urology of the same Institution and were sent in a single formalin filled container. The clinical details of 120 cases were collected by reviewing the medical records. All specimens were processed routinely for $\mathrm{H}$ and $\mathrm{E}$ and IHC studies. After examining the haematoxylin and eosin stained slides, the tumours were classified according to modified Gleason system with ISUP recommendations. Variable degree of AMACR staining was seen in the prostatic carcinoma, which served as a positive control and a benign case was used as a negative control. 120 cases in which AMACR expression was studied were categorised into AMACR positive and negative cases. The factors studied were AMACR expression and mean serum PSA value. The proportion of tumours showing AMACR positivity was expressed as percentage. Statistical analysis was done using SPSS version 16. Chisquare test was used to find if there was any statistically significant association between the levels of serum PSA and histopathological findings studied.

Interpretation of AMACR staining- Positive staining is indicated by brownish cytoplasmic staining of $80-100 \%$ of tumour cells in tissue sections. ${ }^{7}$

\section{RESULTS}

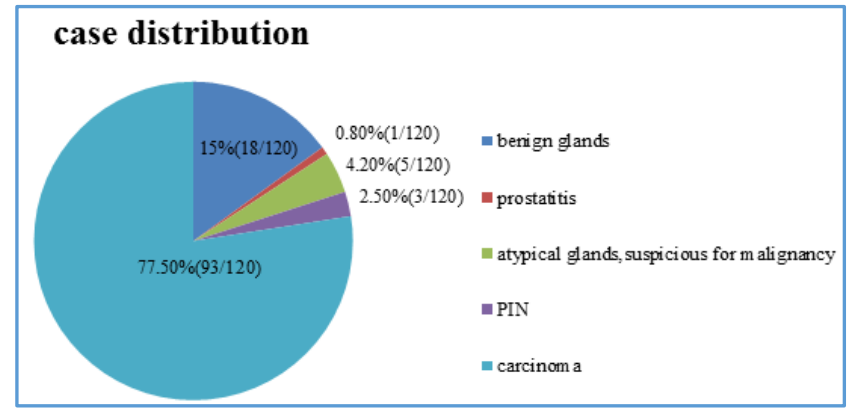

Graph 1. Case Distribution

In this study, majority of cases (93/120 cases; $77.5 \%)$ were diagnosed as prostatic adenocarcinoma.

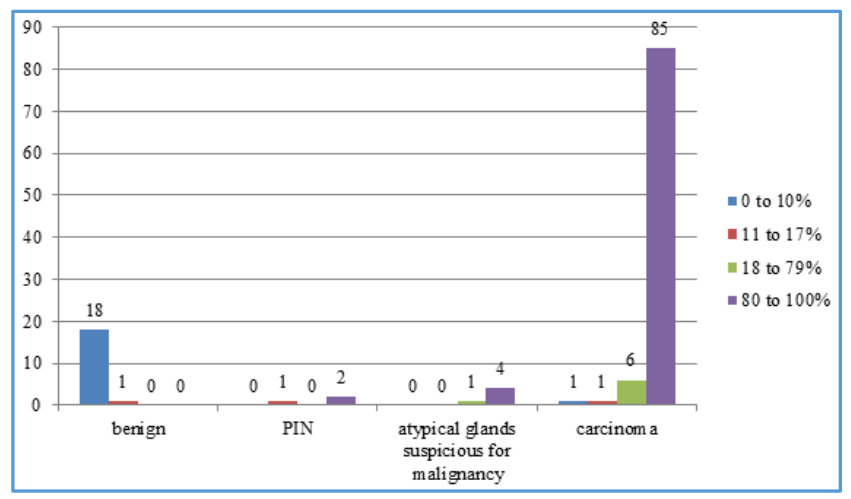
Hyperplasia showing AMACR Negativity

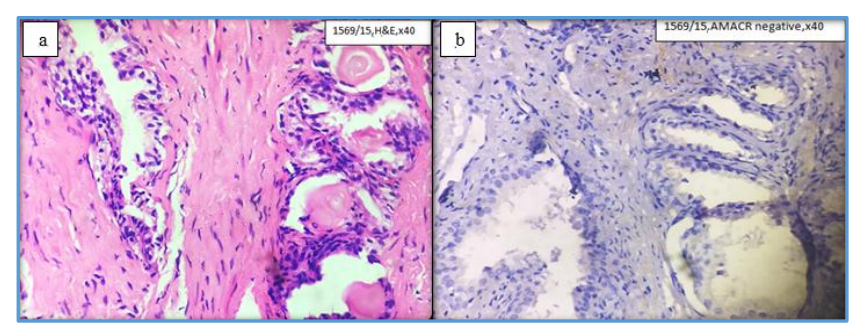

Figure 1. A and B Photomicrograph of Benign Prostatic

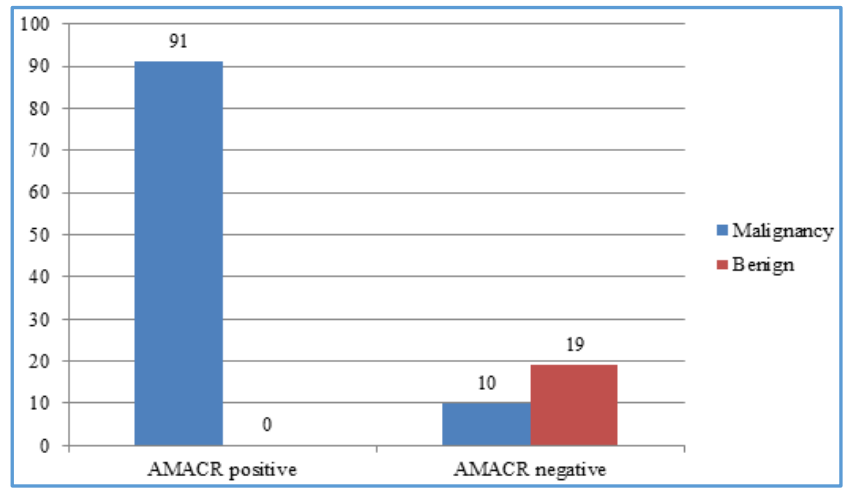

Graph 3. Diagnostic Evaluation of AMACR

In this study $(n=120) 91 \%$ of malignant cases showed AMACR positivity, whereas all benign cases showed AMACR negativity.

Sensitivity and specificity of AMACR in prostatic carcinoma are $90 \%$ and $100 \%$ respectively. Positive predictive value of AMACR is $100 \%$ and negative predictive value of AMACR is $65 \%$.

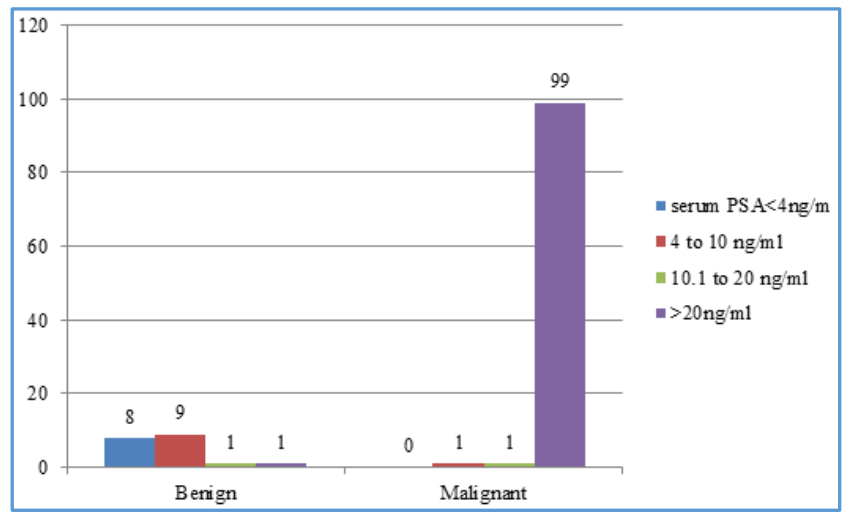

Graph 4. Correlation of H and E Diagnosis with Serum PSA Value

The mean value of serum PSA in malignant cases was $401.54+/-254.429$ and in benign cases was $5.78+/-5.039$. This difference in mean was statistically significant using an independent sample ' $t$ ' test with a ' $t$ ' value of -6.757 and a ' $p$ ' value of 0.001 .

Graph 2. Expression of AMACR in Prostatic Lesions in Present Study 


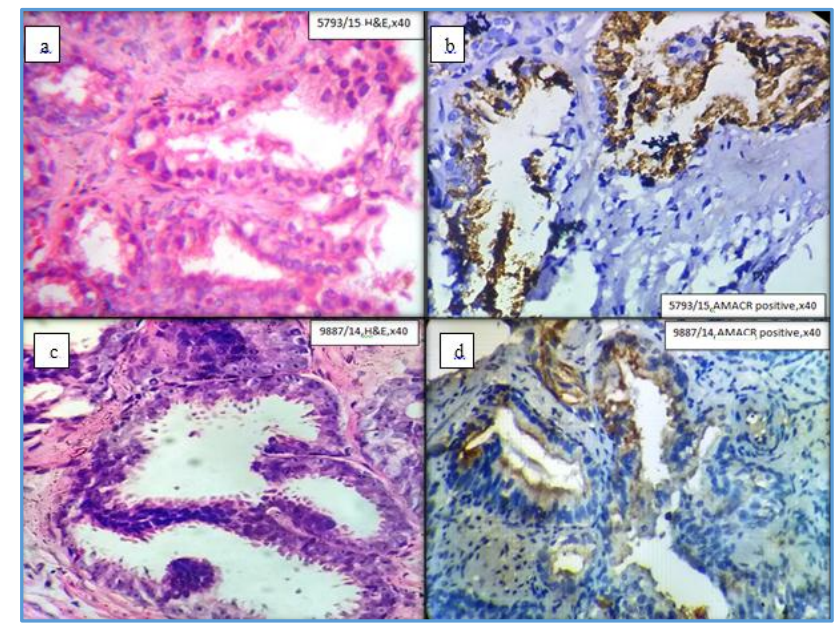

Figure 2. A and B Photomicrograph of Atypical Glands suspicious for Malignancy showing AMACR Positivity. $C$ and D Photomicrograph of PIN showing AMACR Positivity

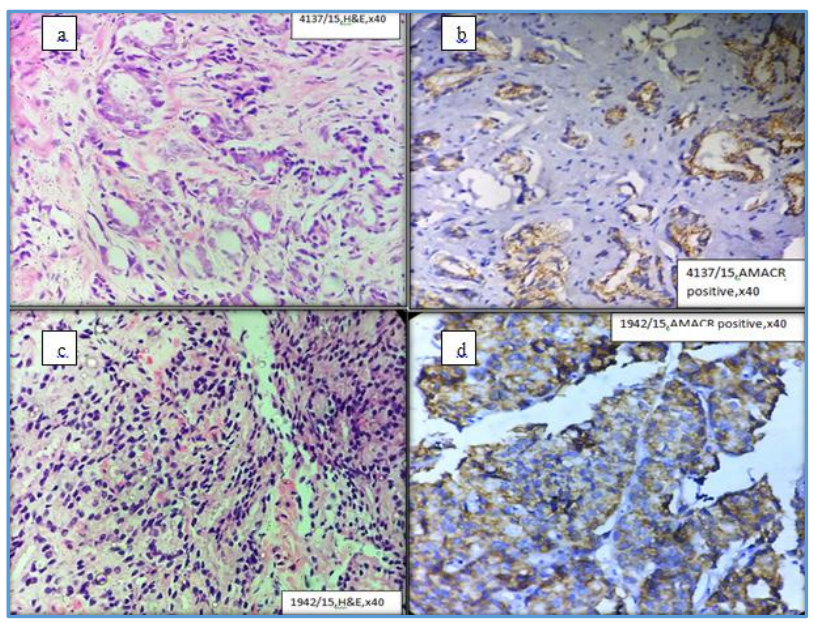

Figure 3. A and B Photomicrograph of Prostatic Carcinoma Gleason Pattern 3 of Small Glands showing AMACR Positivity. C and D Photomicrograph of Prostatic Carcinoma Gleason Pattern 4 of Small Fused Glands showing 100\% AMACR Positivity

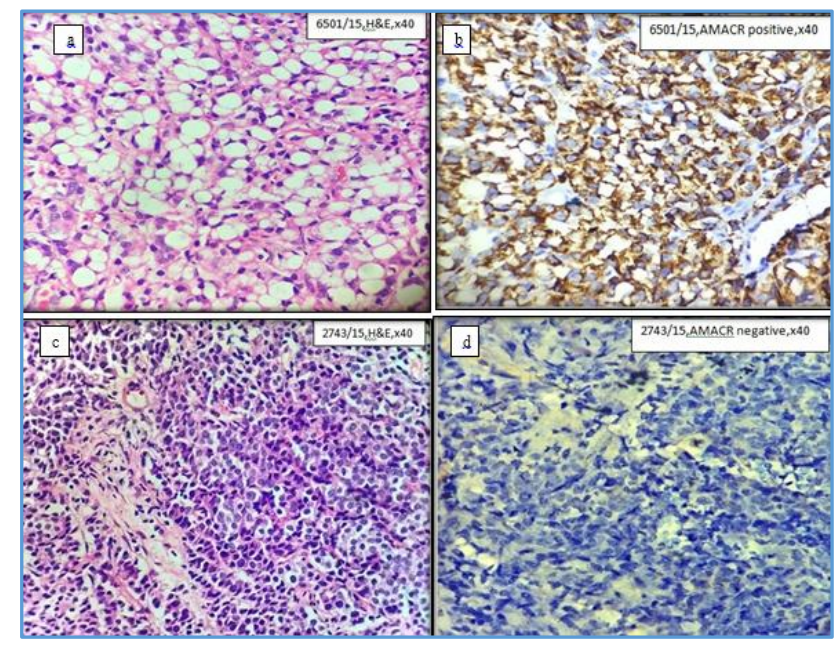

Figure 4. A and B Photomicrograph of Prostatic Carcinoma- Gleason Pattern 5 with Signet Ring Cells showing AMACR Positivity. C and D Photomicrograph of Prostatic Carcinoma- Gleason Pattern 5 with Solid Sheets showing AMACR Negativity

\section{DISCUSSION}

Serum PSA screening and core needle biopsies are the most frequent methods for diagnosing prostatic carcinoma now-adays. Large screening trials have demonstrated that clinically significant carcinomas occur with serum PSA values of 2.5 to $4 \mathrm{mg} / \mathrm{mL}$. So, some experts have proposed lowering the PSA cut-off to $2.5 \mathrm{mg} / \mathrm{mL}$ to improve early detection of carcinoma in young individuals. Hence, serum PSA ${ }^{8}$ measurement is a useful but imperfect cancer screening test with significant rates of false-negative and false-positive results. Evaluation of PSA concentrations after treatment has great value in monitoring progressive or recurrent disease. Ideally, prostatic needle cores for each sextant site should be sent in separate jars so that-1) Atypical site can be preferentially targeted on repeated biopsy; 2) Diagnostic pitfalls (eg, normal anatomic structures like central zone of prostate and seminal vesicles- base biopsy that may mimic high-grade Prostatic Intraepithelial Neoplasia [PIN] or carcinoma, respectively) ${ }^{9}$ can be avoided; 3 ) Will help the pathologist to identify the tumour in the radical prostatectomy specimen when the tumour is extremely focal, especially in $5 \%$ of cases where it may be extremely difficult to identify prostatic carcinoma in radical prostatectomy specimens; 4) With brachytherapy, this helps target areas where extra seeds can be distributed. Hence, biopsy specimens from different areas of the prostate should be submitted to pathology in separate containers.

The Principal Criteria for Diagnosis of well-differentiated Adenocarcinoma by $\mathrm{H}$ and $\mathrm{E}$ Include-

1. A small gland proliferation recognised as being discrete or focally infiltrative on low-power examination,

2. The presence of a single cell lining with complete absence of the basal cell layer,

3. Nucleomegaly, and

4. Presence of large nucleoli. (Nucleoli must be at least 1 micron in diameter).

The rule of "three tools" in low power screening in needle biopsy/TURP specimens are:

(i) Too small glands, (ii) Too crowded glands with back to back arrangement and (iii) Too clear glands.

Evaluating an atypical focus in a needle biopsy of the prostate should be a methodical process. When reviewing needle biopsies one should have a mental balance sheet of one side of the column are features favouring the diagnosis of carcinoma and on the other side of the column are features against the diagnosis of carcinoma.

Wolters $\mathrm{T}$ et $\mathrm{al}^{10}$ in his studies indicated that the overall false negative prostate biopsies (adenocarcinoma/atypical glands suspicious for malignancy diagnosed as benign) were estimated to be $2.4 \%$ to $1.1 \%$ for prostate carcinoma and $1.3 \%$ for atypical suspicious glands.

The modern system for grading adenocarcinoma of prostate is made out from work done by Donald F. Gleason in the 1960s based on a specimen cohort from the Veterans Administration Cooperative Research Group. ${ }^{9}$ Nearly after 50 years the Gleason grading system remains novel, in that it is based on the architectural pattern of the tumour alone (Gleason patterns 1 - 5); the sum of the two most common patterns- that is primary Gleason pattern plus secondary Gleason pattern equals Gleason Score (GS)- conveys the most 
clinical meaning. With the introduction of PSA screening, thin-needle biopsy techniques and expanded sampling over the last two decades it has become necessary for pathologists to diagnose and grade prostatic carcinoma on smaller and better characterised samples.

In 2005, the International Society of Urologic Pathology (ISUP) conducted a conference on Gleason grading to mention emerging issues in the field based on existing data as well as the personal and institutional experience of a large International group of urologic pathologists. The resulting manuscript, "The 2005 International Society of Urological Pathology (ISUP) Consensus Conference on Gleason Grading of Prostatic Carcinoma" serves as a provisional diagnostic guide to modern Gleason grading. ${ }^{11}$

Due to the availability of limited tissue and the presence of many benign mimickers of malignancy (atypical adenomatous hyperplasia, sclerosing adenosis, basal cell hyperplasia, atrophy, seminal vesicle and ejaculatory ducts) in needle biopsy specimens of prostate, diagnosis of carcinoma by $\mathrm{H}$ and $\mathrm{E}$ alone becomes difficult. There are two main indications for use of IHC in prostatic biopsy:

1. In confirming prostate carcinoma and/or distinguishing it from its many benign mimickers, like instances of unusual morphologic patterns of carcinoma or changes due to prior treatment.

2. In distinguishing prostatic carcinoma from non-prostatic malignancies that secondarily involve the prostate. ${ }^{12}$

AMACR, one of the peroxisomal structures, plays a critical role in beta oxidation of branched chain fatty acid. Recently, it has been recognised as a tumour marker for several tumours. (Colorectal, ovarian, breast, bladder, lung and papillary renal cell carcinoma as well as in lymphoma and melanoma) and its role in prostatic carcinogenesis is unknown. By IHC, the majority of prostatic carcinomas $(80 \%-100 \%)$ are positive for AMACR, although a high proportion of high-grade PIN (64\%), some foci of adenosis and some entirely benign glands also show positivity for this marker. ${ }^{13}$

Approximately, $20 \%$ of adenocarcinomas of prostate are AMACR negative, so use of AMACR alone is not helpful for the diagnosis of adenocarcinoma. Thus, AMACR is being used increasingly in conjunction with $\mathrm{H}$ and $\mathrm{E}$ histology and basal cell markers in the workup of difficult prostatic needle biopsy specimens.[8]

To improve the sensitivity and specificity of the diagnosis of adenocarcinoma, a cocktail of two antibodies (p63/p504s) has been used. Combined use of these two antibodies on the same tissue section, p504s as a positive marker and p63 as a negative marker for carcinoma has the potential to improve diagnostic performance. This cocktail is particularly helpful in resolving atypical small acinar proliferations and reduces the percentage of ambiguous diagnosis and the need for additional biopsies. ${ }^{14}$

Additionally, the cocktail is very helpful in highlighting adenocarcinoma associated with high-grade PIN, flat and cribriform high-grade PIN and distorted foci of minimal adenocarcinoma. ${ }^{14}$ AMACR is also a useful diagnostic marker for the adenocarcinoma variants like foamy gland and pseudohyperplastic prostate carcinoma, although positivity is lower than that in acinar adenocarcinoma. ${ }^{15}$

AMACR overexpression has been reported in many other tumours like colorectal, ovarian and breast carcinomas, thus it is not a very useful marker in workup of a metastatic tumour. Hence, positive IHC marker, AMACR was used to assess its sensitivity and specificity. Even after the use of AMACR, a diagnosis of atypical glands suspicious for malignancy was given to certain cases.

120 cases were studied and classified on the basis of morphological features. Of these $93 / 120$ cases $(77.5 \%)$ were diagnosed as carcinoma which comprised 88/93 cases $(94.62 \%)$ of acinar adenocarcinoma, 3/93 cases $(3.22 \%)$ of ductal adenocarcinoma and 2/93 cases of signet ring cell variant of adenocarcinoma; 1/120 diagnosed as a case of prostatitis, 5/120 cases as atypical glands suspicious for malignancy and $3 / 120$ cases as high-grade PIN. This is in accordance with Humphrey PA et al (acinar adenocarcinomaover $90 \%$ of prostatic carcinoma and remaining $5 \%-10 \%$ of carcinoma were of non-acinar type. Among the non-acinar types, ductal adenocarcinoma was most common). ${ }^{16}$

Of $93 / 120$ cases confirmed as carcinoma by $\mathrm{H}$ and $\mathrm{E}, 85$ cases (91.3\%) showed AMACR positivity. Out of 5/120 cases confirmed as atypical glands suspicious for malignancy by $\mathrm{H}$ and E, 4 cases (80\%) showed AMACR positivity. Out of $3 / 120$ cases confirmed as PIN by $\mathrm{H}$ and E, 2 cases (66\%) showed AMACR positivity.

\begin{tabular}{|c|c|c|c|}
\hline $\begin{array}{c}\text { H \& E } \\
\text { Diagnosis }\end{array}$ & $\begin{array}{c}\text { Luo et al17 } \\
\mathbf{n = 1 6 8}\end{array}$ & $\begin{array}{c}\text { Zhou et al6 } \\
\mathbf{n}=\mathbf{3 0 7}\end{array}$ & $\begin{array}{c}\text { Present Study } \\
\mathbf{n = 1 2 0}\end{array}$ \\
\hline Benign Conditions & $<4 \%$ & 4 to $21 \%$ & $0 \%$ \\
\hline Malignancies & $>95 \%$ & $90 \%$ & $91 \%$ \\
\hline \multicolumn{3}{|c|}{ Table 1. Comparison of various Studies } \\
on AMACR Positivity \\
\hline
\end{tabular}

\begin{tabular}{|c|c|c|c|}
\hline Sl. No. & Recent Study & Sensitivity & Specificity \\
\hline 1. & $\begin{array}{c}\text { Kumeresan et al } 18 \\
\mathrm{n}=1034 \text { cases }\end{array}$ & $92 \%$ & $100 \%$ \\
\hline 2. & $\begin{array}{c}\text { Jiang \& Colleagues } \\
\mathrm{n}=361 \text { cases }\end{array}$ & $97 \%$ & $92 \%$ \\
\hline 3. & $\begin{array}{c}\text { Present Study } \\
\mathrm{n}=120 \text { cases }\end{array}$ & $90 \%$ & $100 \%$ \\
\hline \multicolumn{4}{|c|}{ Table 2. Comparison of Sensitivity and Specificity } \\
of AMACR with various Studies \\
\hline
\end{tabular}

The mean serum PSA value in prostatic lesions is statistically significant using an independent sample ' $t$ ' test with a ' $t$ ' value of -6.757 and a ' $p$ ' value of 0.001 .

\section{CONCLUSION}

$\mathrm{H}$ and $\mathrm{E}$ is the gold standard for diagnosing prostatic neoplasms. AMACR helps in excluding the benign mimickers of prostatic carcinoma and in confirming the diagnosis of malignancy by a positive signal rather than loss of signal. Combination of AMACR and basal cell marker, either p63/HMWCK has the advantage of highlighting the presence of malignant cells and absence of basal cell layer. Hence, along with AMACR, basal cell marker helps in confirming the malignancy in morphologically suspicious cases.

Sensitivity and specificity of AMACR in prostatic carcinoma are $90 \%$ and $100 \%$ respectively; $99 \%$ of prostatic carcinoma showed high serum PSA value. 


\section{REFERENCES}

[1] Siegel R, Naishadham D, Jemal A. Cancer statistics, 2012. CA cancer J Clin 2012;62(1):10-29.

[2] Haas GP, Delongchamps N, Brawley OW, et al. The worldwide epidemiology of prostate cancer: perspectives from autopsy studies. The Canadian journal of urology 2008;15(1):3866-71.

[3] Yeole BB. Trends in the prostate cancer incidence in India. Asian Pac J Cancer Prev 2008;9(1):141-4.

[4] Cupp MR, Oesterling JE. Prostate-specific antigen, digital rectal examination, and transrectal ultrasonography: their roles in diagnosing early prostate cancer. Mayo Clin Proc 1993;68(3):297-306.

[5] Molinié V, Hervé JM, Lebret T, et al. Value of the antibody cocktail anti p63 + anti p504s for the diagnosis of prostatic cancer. Ann Pathol 2004;24(1):6-16.

[6] Zhou M, Aydin H, Kanane $\mathrm{H}$, et al. How often does alpha-methyl acyl-CoA-racemase contribute to resolving an atypical diagnosis on prostate needle biopsy beyond that provided by basal cell markers? Am J Surg Pathol 2004;28(2):239-43.

[7] Kunju LP, Rubin MA, Chinnaiyan AM, et al. Diagnostic usefulness of monoclonal antibody P504S in the workup of atypical prostatic glandular proliferations. Am J Clin Pathol 2003;120(5):737-45.

[8] Gjertson CK, Albertsen PC. Use and assessment of PSA in prostate cancer. Med clin North Am 2011;95(1):191-200.

[9] Fine SW, Amin MB, Berney DM, et al. A contemporary update on pathology reporting for prostate cancer: biopsy and radical prostatectomy specimens. European Urology 2012;62(1):20-39.

[10] Wolters T, Kwast THVD, Vissers CJ, et al. Falsenegative prostate needle biopsies: frequency, histopathologic features, and follow-up. Am J Surg Pathol 2010;34(1):35-43.
[11] Epstein JI, Allsbrook WC, Amin MB, et al. The 2005 international society of urological pathology (ISUP) consensus conference on gleason grading of prostatic carcinoma. Am J Surg Pathol 2005;29(9):1228-42.

[12] Osunkoya AO, Netto GJ, Epstein JI. Colorectal adenocarcinoma involving the prostate: report of 9 cases. Hum Pathol 2007;38(12):1836-41.

[13] Jiang Z, Fanger GR, Woda BA, et al. Expression of alpha-methylacyl-CoA racemase (P504S) in various malignant neoplasms and normal tissues: a study of 761 cases. Hum Pathol 2003;34(8):792-6.

[14] Hameed 0, Sublett J, Humphrey PA. Immunohistochemical stains for p63 and alphamethylacyl-CoA racemase, versus a cocktail comprising both, in the diagnosis of prostatic carcinoma: a comparison of the immunohistochemical staining of 430 foci in radical prostatectomy and needle biopsy tissues. Am J Surg Pathol 2005;29(5):579-87.

[15] Zhou M, Jiang Z, Epstein JI. Expression and diagnostic utility of alpha-methylacyl-CoA-racemase (P504S) in foamy gland and pseudohyperplastic prostate cancer. Am J Surg Pathol 2003;27(6):772-8.

[16] Humphrey PA. Variants of prostatic carcinoma in prostate pathology. ASCP Press: Chicago, IL, 2003.

[17] Luo J, Zha S, Gage WR, et al. Alpha-methylacyl-CoA racemase: a new molecular marker for prostate cancer. Cancer Res 2002;62(8):2220-6.

[18] Kumaresan K, Kakkar N, Verma A, et al. Diagnostic utility of alpha-methylacyl CoA racemase (P504S) \& HMWCK in morphologically difficult prostate cancer. Diagnostic Pathology 2010;5:83. 